\title{
Complete solving the quadratic equation mod $2^{n}$
}

\author{
S. M. Dehnavi ${ }^{1}$, M. R. Mirzaee Shamsabad ${ }^{2}$ \\ and A. Mahmoodi Rishakani ${ }^{3}$ \\ ${ }^{1}$ Department of Mathematical and Computer Sciences \\ University of Kharazmi, Tehran, Iran \\ e-mail: dehnavismeipm.ir \\ ${ }^{2}$ Department of Mathematics \\ Shahid Beheshti University, Tehran, Iran \\ e-mail: m_mirzaeedsbu.ac.ir \\ ${ }^{3}$ Department of Sciences \\ Shahid Rajaee Teacher Training University, Tehran, Iran \\ e-mail: am.rishakaniesru.ir
}

Received: 24 December 2017

Accepted: 24 December 2018

Abstract: Quadratic functions have applications in cryptography. In this paper, we investigate the modular quadratic equation

$$
a x^{2}+b x+c=0 \quad\left(\bmod 2^{n}\right),
$$

and provide a complete analysis of it. More precisely, we determine when this equation has a solution and in the case that it has a solution, we give not only the number of solutions, but also the set of solutions, in $O(n)$ time. One of the interesting results of our research is that, if this equation has a solution, then the number of solutions is a power of two. Most notably, as an application, we characterize the number of fixed-points of quadratic permutation polynomials over $\mathbb{Z}_{2^{n}}$, which are used in symmetric cryptography.

Keywords: Quadratic equation mod $2^{n}$, Number of solutions, Set of solutions, Number of fixedpoints, Cryptography.

2010 Mathematics Subject Classification: 11Z05, 14G50. 


\section{Introduction}

The square mapping is one of the tools which is used in cryptography. For instance, the Rabin cryptosystem [6] employs a modular quadratic mapping. As another example, in the design of the stream cipher Rabbit [1], the square map is used. A quadratic polynomial modulo $2^{32}$ is used in the AES finalist block cipher RC6 [5].

The quadratic equation has been solved over various algebraic structures. For example, the quadratic equation over $\mathbb{F}_{2^{n}}$ is solved in Theorem 3.2.15 of [3]. Note that an algorithm for finding the solutions of quadratic equation over $\mathbb{F}_{2^{n}}$ is also given in [8]. This research is not the first one concerning the quadratic equation mod $2^{n}$. For instance, [7] gives the solutions of equation (1) in spacial cases.

In this paper, we examine the quadratic equation $\bmod 2^{n}$. We verify when this equation has a solution and, in the case that it has a solution, we give the number of solutions as well as the set of its solutions in $O(n)$ time. As an application for symmetric cryptography, we characterize the number of fixed-points of quadratic permutation polynomials over $\mathbb{Z}_{2^{n}}$.

In section 2, we give the preliminary notations and definitions. Section 3 is devoted to the main theorems of the paper which solve the modular quadratic equation mod $2^{n}$, completely, and presents its number of solutions along with its set of solutions. In section 4, we conclude the paper.

\section{Notations and definitions}

We denote the well-known ring of integers mod $2^{n}$ by $\mathbb{Z}_{2^{n}}$. For every nonzero element $a \in \mathbb{Z}_{2^{n}}$, we define $p_{2}(a)$ as the greatest power of 2 that divides $a$. The odd part of $a$ or $\frac{a}{2^{p_{2}(a)}}$ is denoted by $o_{2}(a)$, in the current paper. Note that, we define $p_{2}(0):=n$.

The number of elements (cardinal) of a finite set $R$ is denoted by $|R|$. For a function $f: R \rightarrow$ $S$, the preimage of an element $b \in S$ is defined as $\{a \in R \mid b=f(a)\}$ and is denoted by $f^{-1}(b)$. If $f(x)=x$ for some $x \in R$, then $x$ is called a fixed-point of $f$. The $i$-th bit of a natural number $x$ in its binary representation is denoted by $[x]_{i}$. For an integer $j$, we define $e_{j}$ as follows

$$
e_{j}= \begin{cases}0, & j \text { odd } \\ 1, & j \text { even }\end{cases}
$$

A mapping

$$
\begin{gathered}
f: \mathbb{Z}_{2^{n}} \rightarrow \mathbb{Z}_{2^{n}}, \\
f(x)=a x^{2}+b x+c\left(\bmod 2^{n}\right),
\end{gathered}
$$

is called a quadratic polynomial over $\mathbb{Z}_{2^{n}}$. When $f$ is a permutation, it is called a quadratic permutation polynomial over $\mathbb{Z}_{2^{n}}$.

Let $(G, *)$ be a group and $\varphi: G \rightarrow G$ be a group endomorphism. We denote the kernel of $\varphi$ by $\operatorname{ker}(\varphi)$ and the image of $\varphi$ by $\operatorname{Im}(\varphi)$. 


\section{Solving the quadratic equation mod $2^{n}$}

In this section, we study the modular quadratic equation

$$
a x^{2}+b x+c=0 \quad\left(\bmod 2^{n}\right),
$$

and wish to solve it. More precisely, we want to determine:

a) whether (1) has a solution;

b) if it has a solution, then what is the number of its solutions;

c) the set of its solutions.

In the sequel, we note that $x=0$ is equivalent to $p_{2}(x)=n$.

Lemma 3.1. Let $a$, $b$, and $c$ be even and $t=\min \left\{p_{2}(a), p_{2}(b), p_{2}(c)\right\}$. Set $A=\frac{a}{2^{t}}, B=\frac{b}{2^{t}}$, and $C=\frac{c}{2^{t}}$. Consider the equations (1) and

$$
A x^{2}+B x+C=0 \quad\left(\bmod 2^{n-t}\right) .
$$

Let $N_{1}$ and $N_{2}$ be the number of solutions of (1) and (2), respectively. Also, let $\left\{x_{1}, \ldots, x_{N_{2}}\right\}$ be the set of solutions of (2). Then, the set of solutions of (1) is as follows

$$
\left\{x_{i}+r 2^{n-t}: 0 \leq r<2^{t}, 1 \leq i \leq N_{2}\right\} \text {. }
$$

Further, $N_{1}=2^{t} N_{2}$.

Proof. Firstly, fix $1 \leq i \leq N_{2}$ and $0 \leq r<2^{t}$. We show that $x_{i}+r 2^{n-t}$ is a solution of (1):

$$
\begin{aligned}
& a\left(x_{i}+r 2^{n-t}\right)^{2}+b\left(x_{i}+r 2^{n-t}\right)+c \\
& =2^{t} A\left(x_{i}^{2}+r^{2} 2^{2 n-2 t}+x_{i} r 2^{n-t+1}\right)+2^{t} B\left(x_{i}+r 2^{n-t}\right)+2^{t} C \\
& =2^{t} A x_{i}^{2}+A r^{2} 2^{2 n-t}+A x_{i} r 2^{n+1}+B x_{i} 2^{t}+B r 2^{n}+2^{t} C \\
& =2^{t}\left(A x_{i}^{2}+B x_{i}+C\right)=0 \quad\left(\bmod 2^{n}\right) .
\end{aligned}
$$

Conversely, let $x \in \mathbb{Z}_{2^{n}}$ be a solution of (1). Then

$$
2^{t}\left(A x^{2}+B x+C\right)=0\left(\bmod 2^{n}\right) .
$$

So,

$$
A x^{2}+B x+C=0\left(\bmod 2^{n-t}\right) .
$$

One can check that $\chi=x\left(\bmod 2^{n-t}\right)$ is a solution of (2). Thus, all of solutions $y$ of $(1)$ are such that $y=x_{i}+r 2^{n-t}$, for some $1 \leq i \leq N_{2}$ and $0 \leq r<2^{t}$.

Example. Consider the equations

$$
4 x^{2}+4 x+24=0 \quad\left(\bmod 2^{5}\right)
$$

and

$$
x^{2}+x+6=0 \quad\left(\bmod 2^{3}\right) .
$$

The set of solutions of (3) and (4) are $A=\{1,6,9,14,17,22,25,30\}$ and $B=\{1,6\}$, respectively. One can check that Lemma 3.1 holds for this example and $|A|=2^{2}|B|$.

The proof of the following lemma is straightforward. 
Lemma 3.2. The equation (1) has no solutions when $p_{2}(a)=p_{2}(b)=p_{2}(c)=0$ or when $p_{2}(a)>0, p_{2}(b)>0$, and $p_{2}(c)=0$.

Lemma 3.3. If $p_{2}(a)>0$ and $p_{2}(b)=0$, then the equation (1) has a unique solution.

Proof. Consider the two following cases:

Case I) Let $p_{2}(a)>0, p_{2}(b)=p_{2}(c)=0, a=2 A, b=2 B+1$, and $c=2 C+1$. In this case, any solution $x$ of (1) is odd; so, we have $x=2 X+1$. Thus, we have

$$
2 A(2 X+1)^{2}+(2 B+1)(2 X+1)+2 C+1=0 \quad\left(\bmod 2^{n}\right),
$$

which simplifies to

$$
4 A X^{2}+(4 A+2 B+1) X+A+B+C+1=0 \quad\left(\bmod 2^{n-1}\right) .
$$

So, if we set $\alpha=4 A, \beta=4 A+2 B+1$, and $\gamma=A+B+C+1$, then $[x]_{0}=1$ and we must solve the equation

$$
\alpha X^{2}+\beta X+\gamma=0 \quad\left(\bmod 2^{n-1}\right),
$$

such that $p_{2}(\alpha)>0$ and $p_{2}(\beta)=0$. Now, we have either $p_{2}(\gamma)=0$, which is this same case or $p_{2}(\gamma)>0$, which is Case II, below.

Case II) Let $p_{2}(a)>0, p_{2}(c)>0, p_{2}(b)=0, a=2 A, b=2 B+1$, and $c=2 C$. In this case, $x=2 X$. So we have

$$
2 A(2 X)^{2}+(2 B+1)(2 X)+2 C=0 \quad\left(\bmod 2^{n}\right)
$$

or

$$
4 A X^{2}+(2 B+1) X+C=0 \quad\left(\bmod 2^{n-1}\right) .
$$

Put $\alpha=4 A, \beta=2 B+1$, and $\gamma=C$. Then $[x]_{0}=0$ and we should solve the equation

$$
\alpha X^{2}+\beta X+\gamma=0 \quad\left(\bmod 2^{n-1}\right),
$$

with $p_{2}(\alpha)>0$ and $p_{2}(\beta)=0$. Now, if $p_{2}(\gamma)=0$, then we transit to Case I and if $p_{2}(\gamma)>0$, then we transit to this same case. Therefore, (1) has a unique solution.

The trend of the proof of Lemma 3.3 justifies the correctness of Algorithm 1, which computes the solution of (1) with the conditions of Lemma 3.3 in $O(n)$ time.

Algorithm 1: $\operatorname{Solve}(a, b, c, n)$

Input: $a, b, c \in \mathbb{Z}_{2^{n}}$ with $p_{2}(a)>0$ and $p_{2}(b)=0$.

Output: The solution of (1) in binary form.

for $i=0$ to $n-1$ do

begin

if $p_{2}(c)>0$ then

$[x]_{i}=0$

Solve $\left(2 a, b, \frac{c}{2}, n-1\right)$

else

$[x]_{i}=1$

Solve $\left(2 a, 2 a+b, \frac{a}{2}+\left\lfloor\frac{b}{2}\right\rfloor+\left\lfloor\frac{c}{2}\right\rfloor+1, n-1\right)$. 
Lemma 3.4. In the case that $p_{2}(a)=p_{2}(b)=0$ and $p_{2}(c)>0$, the equation (1) has two solutions.

Proof. Consider the equation $2 a y^{2}+b y+\frac{c}{2}=0 \quad\left(\bmod 2^{n-1}\right)$. Lemma 3.3 shows that this equation has a unique solution $\delta \in \mathbb{Z}_{2^{n-1}}$. One can check that $\pi=2 \delta$ is a solution of (1), in this case. On the other hand, Lemma 3.3 shows that the equation

$$
2 a z^{2}+(2 a+b) z+\frac{a+b+c}{2}=0 \quad\left(\bmod 2^{n-1}\right)
$$

has a unique solution $\rho \in \mathbb{Z}_{2^{n-1}}$. It is straightforward to see that $\varepsilon=2 \rho+1$ is a solution of (1) in $\mathbb{Z}_{2^{n}}$. Now, we show that (1) has no other solutions. Suppose that $x$ is a solution of (1). We have the two following cases:

Case I) $x=2 X$; we have

$$
4 a X^{2}+2 b X+c=0 \quad\left(\bmod 2^{n}\right)
$$

So,

$$
2 a X^{2}+b X+\frac{c}{2}=0 \quad\left(\bmod 2^{n-1}\right)
$$

which is not a new solution.

Case II) $x=2 X+1$; in this case we have

$$
4 a X^{2}+(4 a+2 b) X+a+b+c=0 \quad\left(\bmod 2^{n}\right) .
$$

So,

$$
2 a X^{2}+(2 a+b) X+\frac{a+b+c}{2}=0 \quad\left(\bmod 2^{n-1}\right),
$$

which is not a new solution.

The proof of next lemma is straightforward.

Lemma 3.5. If $a$ is an odd element in $\mathbb{Z}_{2^{n}}$, then $a^{2}=1(\bmod 8)$.

The next theorem provides the set of solutions of the equation $x^{2}=a\left(\bmod 2^{n}\right)$.

Theorem 3.6. Suppose that $f: \mathbb{Z}_{2^{n}} \rightarrow \mathbb{Z}_{2^{n}}$ is defined as $f(x)=x^{2}\left(\bmod 2^{n}\right)$. Then,

a) For the three cases $p_{2}(a)=n, p_{2}(a)=n-1$ with $e_{n}=0$, and $a=2^{n-2}$ with $e_{n}=1$, we have

$$
\left|f^{-1}(a)\right|=2^{\frac{n-1+e_{n}}{2}} .
$$

b) For the two cases $p_{2}(a)=1(\bmod 2)$, and $p_{2}(a)=0(\bmod 2)$ with $0 \leq p_{2}(a) \leq n-3$ and $o_{2}(a) \neq 1(\bmod 8)$, we have

$$
\left|f^{-1}(a)\right|=0 \text {. }
$$

c) For the case of $p_{2}(a)=0(\bmod 2)$ with $0 \leq p_{2}(a) \leq n-3$ and $o_{2}(a)=1(\bmod 8)$, we have

$$
\left|f^{-1}(a)\right|=2^{\frac{p_{2}(a)+4}{2}} .
$$


Proof. Case a) On one hand, every $a \in \mathbb{Z}_{2^{n}}$ with $p_{2}(a) \geq\left\lceil\frac{n}{2}\right\rceil$ satisfies $x^{2}=0\left(\bmod 2^{n}\right)$. So, $\left|f^{-1}(a)\right|$ is at least $2^{n-\left\lceil\frac{n}{2}\right\rceil}=2^{\frac{n-1+e_{n}}{2}}$. On the other hand, for each $a \in \mathbb{Z}_{2^{n}}$ with $p_{2}(a)<\left\lceil\frac{n}{2}\right\rceil$, $a^{2} \neq 0\left(\bmod 2^{n}\right)$. Thus, $\left|f^{-1}(a)\right|=2^{\frac{n-1+e_{n}}{2}}$.

Now, suppose that $n$ is odd and $p_{2}(a)=n-1$; i.e., $a=2^{n-1}$. Let $x=2^{r} q$ with odd $q$. We have

$$
2^{2 r} q^{2}=2^{n-1}\left(\bmod 2^{n}\right) .
$$

So, $r=\frac{n-1}{2}, 1 \leq q \leq 2^{\frac{n+1}{2}}-1$ and $q^{2}=1(\bmod 2)$. Thus, only the odd $q$ 's satisfy the equation $x^{2}=2^{n-1}\left(\bmod 2^{n}\right)$. Therefore, $\left|f^{-1}(a)\right|=2^{\frac{n-1+e_{n}}{2}}$.

Now, let $n$ be even and $p_{2}(a)=n-2$. So, $a=s 2^{n-2}$, where $s \in\{1,3\}$. If $s=1$, put $x=2^{r} q$ with odd $q$. Then

$$
2^{2 r} q^{2}=2^{n-2}\left(\bmod 2^{n}\right) .
$$

Hence $r=\frac{n-2}{2}, 1 \leq q \leq 2^{\frac{n+2}{2}}-1$ and $q^{2}=1(\bmod 4)$. Thus, only half of odd $q$ 's satisfy the equation $x^{2}=2^{n-1}\left(\bmod 2^{n}\right)$. Therefore, $\left|f^{-1}(a)\right|=2^{\frac{n-1+e_{n}}{2}}$.

Case b) In the proof of the Case a), put $s=3$. Consider the equation $x^{2}=2^{n-2} \times 3\left(\bmod 2^{n}\right)$ and suppose that $x=2^{r} q$ with odd $q$. Then,

$$
2^{2 r} q^{2}=2^{n-2} \times 3\left(\bmod 2^{n}\right) .
$$

So, $r=\frac{n-2}{2}$ and $q^{2}=3(\bmod 4)$. Thus, by Lemma 3.5, we have $\left|f^{-1}(a)\right|=0$.

Now, suppose that $p_{2}(a)=1(\bmod 2)$. Since the square of any odd element is odd, so only even elements $x \in \mathbb{Z}_{2^{n}}$ can satisfy $x^{2}=a\left(\bmod 2^{n}\right)$. Let $x=2^{r} q, r \neq 0$, and suppose that $q$ is odd. Then $p_{2}\left(x^{2}\right)=2 r$ which contradicts $p_{2}(a)=1(\bmod 2)$. Therefore, $\left|f^{-1}(a)\right|=0$.

Now, let $p_{2}(a)=0(\bmod 2)$ and $o_{2}(a) \neq 1(\bmod 8)$. So, $a=2^{2 j} t$, where $p_{2}(a)=2 j$ and $t=o_{2}(a)$. If $x=2^{r} q$ with odd $q$, then

$$
2^{2 r} q^{2}=2^{2 j} t\left(\bmod 2^{n}\right)
$$

Consequently, $r=j$ and $q^{2}=t\left(\bmod 2^{n-2 j}\right)$. Thus, regarding Lemma 3.5, $\left|f^{-1}(a)\right|=0$.

Case c) We use Theorem 13.3 in [2] to prove this case. Suppose that $p_{2}(a)=0$ and $a=$ $1(\bmod 8)$. The algebraic structure $(G, *)$, where $G$ is the subset of odd elements in $\mathbb{Z}_{2^{n}}$ and $*$ is the operator of multiplication modulo $2^{n}$ is a group structure. The function $\phi: G \rightarrow G$ with $\phi(g)=g * g$ is a group endomorphism on $G$. To compute $|k e r(\phi)|$, we must count the number of solutions for the equation $x * x=1_{G}$. In other words, we must count the number of solutions for the equation $x^{2}=1\left(\bmod 2^{n}\right)$. We have

$$
(x-1)(x+1)=0\left(\bmod 2^{n}\right) .
$$

Since $x$ is odd, so for some $q \in \mathbb{Z}_{2^{n}}, x=2 q+1\left(\bmod 2^{n}\right)$. So,

$$
4 q(q+1)=0\left(\bmod 2^{n}\right) .
$$

Consequently, $q=0, q=2^{n-2}, q=2^{n-1}, q+1=2^{n-2}, q+1=2^{n-1}$. Substituting the values of $q$, we have the solutions $x_{1}=1, x_{2}=2^{n}-1, x_{3}=2^{n-1}+1$, and $x_{4}=2^{n-1}-1$. Thus $|\operatorname{ker}(\phi)|=4$ and since $|\operatorname{Im}(\phi)|=\frac{|G|}{|\operatorname{ker}(\phi)|}$, we have

$$
|\operatorname{Im}(\phi)|=\frac{2^{n-1}}{4}=2^{n-3} .
$$




\begin{tabular}{|c|c|c|}
\hline \hline Conditions & Verified in & Number of solutions \\
\hline \hline $\begin{array}{c}p_{2}(a)>0, p_{2}(b)>0, p_{2}(c)>0 \\
t=\min \left\{p_{2}(a), p_{2}(b), p_{2}(c)\right\}\end{array}$ & Lemma 3.1 & $\begin{array}{c}2^{t} \text { times the number of solutions } \\
\text { of a corresponding other case }\end{array}$ \\
\hline$p_{2}(a)=0, p_{2}(b)=0, p_{2}(c)=0$ & Lemma 3.2 & 0 \\
\hline$p_{2}(a)>0, p_{2}(b)>0, p_{2}(c)=0$ & Lemma 3.2 & 0 \\
\hline$p_{2}(a)>0, p_{2}(b)=0, p_{2}(c)=0$ & Lemma 3.3 & 1 \\
\hline$p_{2}(a)>0, p_{2}(b)=0, p_{2}(c)>0$ & Lemma 3.3 & 1 \\
\hline$p_{2}(a)=0, p_{2}(b)=0, p_{2}(c)>0$ & Lemma 3.4 & 2 \\
\hline $\begin{array}{l}p_{2}(a)=0, p_{2}(b)>0, p_{2}(c)=0 \\
b=2 B, s=a^{-2} B^{2}-a^{-1} c, r=p_{2}(s)\end{array}$ & Corollary 3.6.1 & $\begin{array}{c}0 \text { in some cases } \\
\text { and } 2^{\frac{r}{2}+2} \text { o.w. }\end{array}$ \\
\hline $\begin{array}{l}p_{2}(a)=0, p_{2}(b)>0, p_{2}(c)>0 \\
b=2 B, s=a^{-2} B^{2}-a^{-1} c, r=p_{2}(s)\end{array}$ & Corollary 3.6.1 & $\begin{array}{c}0 \text { in some cases } \\
\text { and } 2^{\frac{r}{2}+2} \text { o.w. }\end{array}$ \\
\hline \hline
\end{tabular}

Table 1. The summary of cases of solving equation (1)

On the other hand, according to Lemma 3.5 and since the number of elements in $\mathbb{Z}_{2^{n}}$ in the form of $8 q+1$ is equal to $2^{n-3}$ and $|\operatorname{Im}(\phi)|=2^{n-3}$, so every element in the form of $8 q+1$ in $\mathbb{Z}_{2^{n}}$ is a square. Thus, the equation $x^{2}=a\left(\bmod 2^{n}\right)$ has at least one solution. Obviously this solution, say $x$, is odd: $x=2 y+1$. So we have $(2 y+1)^{2}=8 q+1$ or $y^{2}+y-2 q=0$, for some $q$. By Lemma 3.4, this equation has two solutions $q_{1}$ and $q_{2}$. One can check that $q_{3}=2^{n}-q_{1}$ and $q_{4}=2^{n}-q_{2}$ are the two other solutions. Consequently,

$$
\left|f^{-1}(a)\right|=|k e r(\phi)|=4=2^{\frac{p_{2}(a)+4}{2}} .
$$

Now, suppose that $p_{2}(a)=0(\bmod 2), 2 \leq p_{2}(a) \leq n-3$ and $o_{2}(a)=1(\bmod 8)$. In this case, we have $a=2^{2 j} t$ with $p_{2}(a)=2 j$ and $t=o_{2}(a)$. Let $x=2^{r} q$ with odd $q$. Then,

$$
2^{2 r} q^{2}=2^{2 j} t\left(\bmod 2^{n}\right)
$$

So, $r=j$ and $q^{2}=t\left(\bmod 2^{n-2 j}\right)$. Regarding Lemma 3.5 and the proof of Case b), this equation has four solutions $q_{1}, q_{2}, q_{3}, q_{4}$ with $0 \leq q_{i} \leq 2^{n-2 j}-1$. For each of these solutions, we present $2^{j}$ solutions

$$
x_{s, t}=2^{j}\left(s 2^{n-2 j+1}+q_{t}\right), \quad 0 \leq s<2^{j}, \quad 1 \leq t \leq 4 .
$$

We have,

$$
\begin{aligned}
x_{s, t}^{2} & =2^{2 j}\left(s^{2} 2^{2 n-4 j+2}+q_{t}^{2}+2 s 2^{n-2 j+1}\right) \\
& =s^{2} 2^{2 n-2 j+2}+2^{2 j} q_{t}^{2}+s 2^{n+2} \\
& =2^{2 j} q_{t}^{2}\left(\bmod 2^{n}\right) .
\end{aligned}
$$

Regarding $2 j \leq n-3$, we have $2 n-2 j \geq n+3$. Therefore,

$$
\left|f^{-1}(a)\right|=2^{\frac{p_{2}(a)+4}{2}} \text {. }
$$

Note that Theorem 3.6 gives the set of solutions that are needed in the next corollary. 
Corollary 3.6.1. Let $p_{2}(a)=0, p_{2}(b)>0$, and $b=2 B$. Put $s=a^{-2} B^{2}-a^{-1} c, r=p_{2}(s)$, and $q=o_{2}(s)$. If $p_{2}(r)=0$ or $q \neq 1(\bmod 8)$, then (1) has no solutions. Otherwise, (1) has $2^{\frac{r}{2}+2}$ solutions.

Proof. We have

$$
a x^{2}+2 B x+c=0 \quad\left(\bmod 2^{n}\right)
$$

or

$$
x^{2}+2 a^{-1} B x+a^{-1} c=0 \quad\left(\bmod 2^{n}\right) .
$$

So, we get

$$
\left(x+a^{-1} B\right)^{2}=s \quad\left(\bmod 2^{n}\right) .
$$

Now, by Theorem 3.6, if $p_{2}(r)=0$ or $q \neq 1(\bmod 8)$, then (1) has no solutions and, otherwise, it has $2^{\frac{r}{2}+2}$ solutions.

In Corollary 3.6.1, one should note that if $p_{2}(c)=2\left(p_{2}(b)-1\right)$ or $p_{2}(c)>2\left(p_{2}(b)-1\right)$ with $p_{2}\left(p_{2}(c)\right)=0$, then equation (1) has no solutions. In Corollary 3.6.1, if $p_{2}(c)<2\left(p_{2}(b)-1\right)$ or $p_{2}(c)>2\left(p_{2}(b)-1\right)$ with $p_{2}\left(p_{2}(c)\right)>0$, then we should compute $s(\bmod 8)$. The interesting point is that, since $a^{-2}=1(\bmod 8)$, it suffices to compute $S=B^{2}-a^{-1} c$.

It is a well-known fact that (see [4] for example) a polynomial $a x^{2}+b x+c$ over $\mathbb{Z}_{2^{n}}$ is a permutation polynomial, iff $p_{2}(a)>0$ and $p_{2}(b)=0$. Lemma 3.3 provides another proof of this fact. The number of fixed-points, is one of the properties which is studied in symmetric cryptography. The less is the number of fixed-points, the stronger is the component, from this aspect. In the next corollary, we characterize the number of fixed-points for quadratic permutation polynomials over $\mathbb{Z}_{2^{n}}$.

Corollary 3.6.2. Suppose that $f(x)=a x^{2}+b x+c$ on $\mathbb{Z}_{2^{n}}$ is a permutation polynomial; i.e., $p_{2}(a)>0$ and $p_{2}(b)=0$. Obviously, the number of fixed-points of $f$ is equal to the number of solutions for $a x^{2}+(b-1) x+c=0,\left(\bmod 2^{n}\right)$. So, regarding Table $1, f$ has no fixed-points (the best case, from the viewpoint of cryptography) if $p_{2}(c)=0$. Otherwise, it has $2^{t}$ fixed-points, for some $t \geq 1$, if it has any.

\section{Conclusion}

Quadratic functions have applications in cryptography. In this paper, we study the quadratic equation mod $2^{n}$. We determine whether this equation has a solution or not and in the case that it has a solution, we give the number of solutions along with the set of its solutions in $O(n)$ time.

One of our results is the fact that, when the quadratic equation modulo a power of two has a solution, then the number of its solutions is a power of two. The other interesting application is characterizing the number of fixed-points of a quadratic permutation polynomial over $\mathbb{Z}_{2^{n}}$. 


\section{References}

[1] Boesgaard, M., Vesterager, M., \& Zenner, E. (2008). The Rabbit Stream Cipher. New Stream Cipher Designs - The eSTREAM Finalists, 69-83.

[2] Fraleigh, J. B. (1999). A First Course in Abstract Algebra. Sixth edition, Addison-Wesley Publishing Company Inc.

[3] Goresky, M., \& Klapper, A. (2005). Algebraic Shift Register Sequences. Cambridge University Press.

[4] Rivest, R. L. (2001). Permutatin Polynomials modulo $2^{w}$. Finite Fields and Their Apllication, 7, 287-292.

[5] Rivest, R. L., Robshaw, M. J. B., Sidney, R., \& Yin, Y. L. (1998). The RC6 Block Cipher. M.I.T., RSA Laboratories.

[6] Stinson, D. R. (2003). Cryptography - Theory and Practice. 3rd edn. Chapman and Hall/CRC, Boca Raton.

[7] Vincent, C. (2017). Notes on solving $x^{2}=a(\bmod n)$, Available online: https: //www.uvm.edu/ cvincen1/files/teaching/spring2017-math255/ quadraticequation.pdf.

[8] Pommerening, K. (2000). Quadratic Equation in Finite Fields of Characteristic 2. English version (February 2012), Available online: http: / / www.klauspommerening.de/ MathMisc/QuGlChar2.pdf. 